\title{
Design and development of a 329-segment tip-tilt piston mirror array for space-based adaptive optics
}

\author{
Jason B. Stewart ${ }^{* a}$, Thomas G. Bifano ${ }^{\mathrm{b}, \mathrm{c}}$, Paul Bierden ${ }^{\mathrm{c}}$, Steven Cornelissen ${ }^{\mathrm{c}}$, \\ Timothy Cook ${ }^{\mathrm{d}}$, B. Martin Levine ${ }^{\mathrm{e}}$ \\ ${ }^{a}$ Electrical and Computer Engineering Department, Boston University; ${ }^{b}$ Manufacturing Engineering Department, Boston \\ University; ${ }^{\mathrm{c}}$ Boston Micromachines Corporation; ${ }^{\mathrm{d}}$ Astronomy Department, Boston University; ${ }^{\mathrm{e}} \mathrm{Jet}$ Propulsion \\ Laboratory, California Institute of Technology
}

\begin{abstract}
We report on the development of a new MEMS deformable mirror (DM) system for the hyper-contrast visible nulling coronagraph architecture designed by the Jet Propulsion Laboratory for NASA's Terrestrial Planet Finding (TPF) mission. The new DM is based largely upon existing lightweight, low power MEMS DM technology at Boston University (BU), tailored to the rigorous optical and mechanical requirements of the nulling coronagraph. It consists of 329-hexagonal segments on a 600I m pitch, each with tip/tilt and piston degrees of freedom. The mirror segments have $1 \mathrm{I} \mathrm{m}$ of stroke, a tip/tilt range of 600 arc-seconds, and maintain their figure to within $2 \mathrm{~nm}$ RMS under actuation. The polished polycrystalline silicon mirror segments have a surface roughness of 5nm RMS and an average curvature of $270 \mathrm{~mm}$. Designing a mirror segment that maintains its figure during actuation was a very significant challenge faced during DM development. Two design concepts were pursued in parallel to address this challenge. The first design uses a thick, epitaxial grown polysilicon mirror layer to add rigidity to the mirror segment. The second design reduces mirror surface bending by decoupling actuator diaphragm motion from the mirror surface motion. This is done using flexure cuts around the mirror post in the actuator diaphragm. Both DM architectures and their polysilicon microfabrication process are presented. Recent optical and electromechanical characterization results will also be discussed, in addition to plans for further improvement of DM figure to satisfy nulling coronagraph optical requirements.
\end{abstract}

\section{INTRODUCTION}

The NASA TPF mission seeks to optically detect, characterize and study Earth-like extrasolar planets located in the habitable zones of nearby stars. In an effort to determine if these planets are capable of harboring life, the light reflected and emitted from these planets will be captured and examined by two observatories based in space, one operating in the infrared and the other in the visible. In order to improve the contrast ratio of light detected from the planet, both observatories are faced with the non-trivial challenge of suppressing starlight emitted from the parent star ${ }^{1}$.

The new BU DM presented here was developed for the visible nulling coronagraph instrument, which is a competing technology for the TPF Coronagraphic Imaging Observatory. The nulling coronagraph is a Mach-Zehnder style shearing interferometer coupled with a coherent, fiber optic spatial filter array that can be used behind a single aperture telescope to enhance the contrast ratio of planet light to the level necessary for detection. A candidate star that is located on the telescope axis is destructively interfered by the nuller, while planet light that is off-axis passes through the coronagraph optics for detection. For successful planet imaging, parent starlight must be suppressed by a factor of $10^{-10}$, which is challenging on many levels. To achieve this contrast ratio, the coronagraph optical system requires a unique DM design that performs conventional wavefront phase correction, but also has a tip/tilt feature to control sub-aperture coupling to the fiber optic spatial filter. The success of the interferometer and spatial filter combination to produce nulls to the level necessary for planet detection strongly depends on DM performance ${ }^{2,3}$.

Wavefront aberrations in space-based stellar observatories are slowly varying, and created by imperfect optics, shifts in telescope alignment during launch, and thermal fluctuations during observation. Wavefront errors of this nature do not necessarily require a great deal of stroke for correction (tip, tilt and focus terms can be removed with other optical elements). For the nulling coronagraph it is more essential to have high precision positioning resolution, nanometer level stability, and hysteresis-free motion, all of which are advantages of an electrostatically controlled MEMS DM. For

*jstew@bu.edu, (617) 353-9961, 8 Saint Mary’s St, Boston, MA 02215

MEMS/MOEMS Components and Their Applications III, edited by Scot S. Olivier, Srinivas A. Tadigadapa, Albert K. Henning,

Proc. of SPIE Vol. 6113, 61130O, (2006) · 0277-786X/06/\$15 - doi: 10.1117/12.651373 
successful implementation into the nulling coronagraph, the new DM must have a positioning resolution of $0.1 \mathrm{~nm}$ piston motion and 0.06 arc-seconds tip/tilt. Achieving this resolution and stability is largely dependent on high voltage driver design, which will not be addressed here. Previously developed segmented BU DM technology, which provided the foundation for this new design has demonstrated nanometer-level repeatability and is absent of hysteresis. Lastly, to meet coronagraph specifications, the optical quality for each DM segment must have a surface roughness less than $10 \mathrm{~nm}$ RMS and a radius of curvature greater than $5 \mathrm{~m}$. The mirror must also maintain this shape to within 10nm RMS during actuation. These three items present the primary technical challenges faced by the new DM design.

The first section of this paper introduces the design concepts developed for meeting/exceeding nulling coronagraph specifications, and the mirror microfabrication process. The second section presents the optical and electromechanical characterization results for devices fabricated thus far using both design concepts.

\section{THE DEVICE}

The new nulling coronagraph DM architecture (referred to hereafter as the TPF DM) is based largely upon existing MEMS DM technology at Boston University, tailored to the requirements of the visible nulling coronagraph. Existing DMs are fabricated using a three-layer polycrystalline silicon surface micromachining process that uses silicon-oxide as a sacrificial material. Both segmented and continuous mirrors exist and are controlled by surface-normal electrostatic actuators (Figure 1). The actuators are comprised of a compliant electrode diaphragm ( $2^{\text {nd }}$ polysilicon layer) supported along two edges above a fixed electrode ( $1^{\text {st }}$ polysilicon layer). The top surface of the actuator diaphragm is connected to the mirror surface $\left(3^{\text {rd }}\right.$ polysilicon layer) by a post at its center.

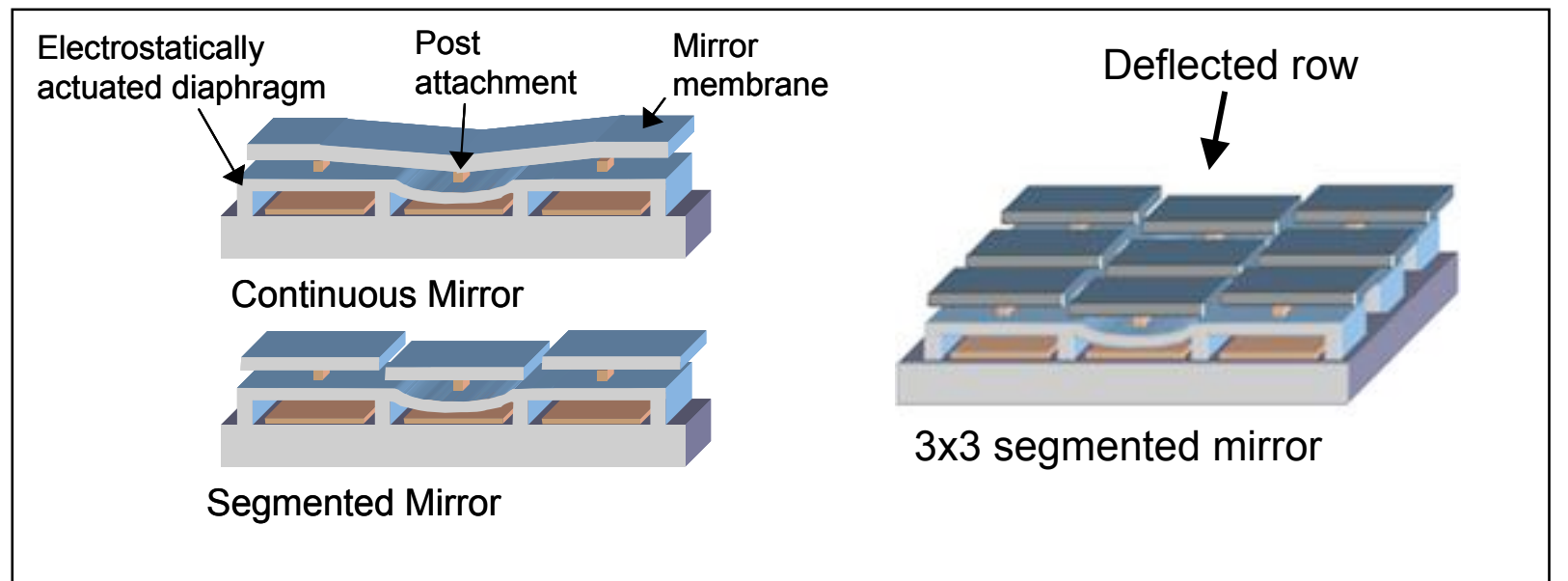

Figure 1: Cross sectional schematic of a segmented and continuous conventional BU MEMS DM showing three actuators (left). Cross sectional schematic of a 3x3 segmented DM with a deflected column of actuators (right).

Existing BU DM designs have several microns of stroke, no hysteresis, surface flatness typically below 25nm RMS, and reflectivity enhanced by aluminum or gold coatings on the final polysilicon layer. They consume very little power due to the small actuator capacitance $(\sim 100 \mathrm{fF})$ and can be operated at frame rates up to $7 \mathrm{kHz}^{4,5}$.

To adapt the above architecture to the TPF DM, the actuator geometry and microfabrication process were modified. A schematic and summary for the new DM design can be seen in Figure 2. It consists of 329 hexagonal mirror segments, each supported by three independent electrostatic actuators from two different actuator rows. The three actuators are connected to the hexagonal mirror segment via posts that resemble vertices of an equilateral triangle. These actuators are identical to those exhibited in Figure 1, but every other row is now offset by a length equal to half the actuator span. This actuator geometry provides the mirror with unlimited degrees of tip/tilt motion, and when the actuators are deflected by equal amounts, the mirror segment can be moved in a piston motion. The mirror segments are designed to have 1I $\mathrm{m}$ of piston stroke when they are tilted to $3 \mathrm{mrad}$. In other words, if the mirror elements remained flat, the mirror could experience 2I $\mathrm{m}$ of piston motion before reaching the actuator limit. However, $1 \mathrm{I} \mathrm{m}$ of this motion is reserved for tip/tilt behavior. Lastly, the mirror segments are 600I $\mathrm{m}$ in their longest dimension, creating a DM aperture of $9.5 \mathrm{~mm}$ by $12 \mathrm{~mm}$. 


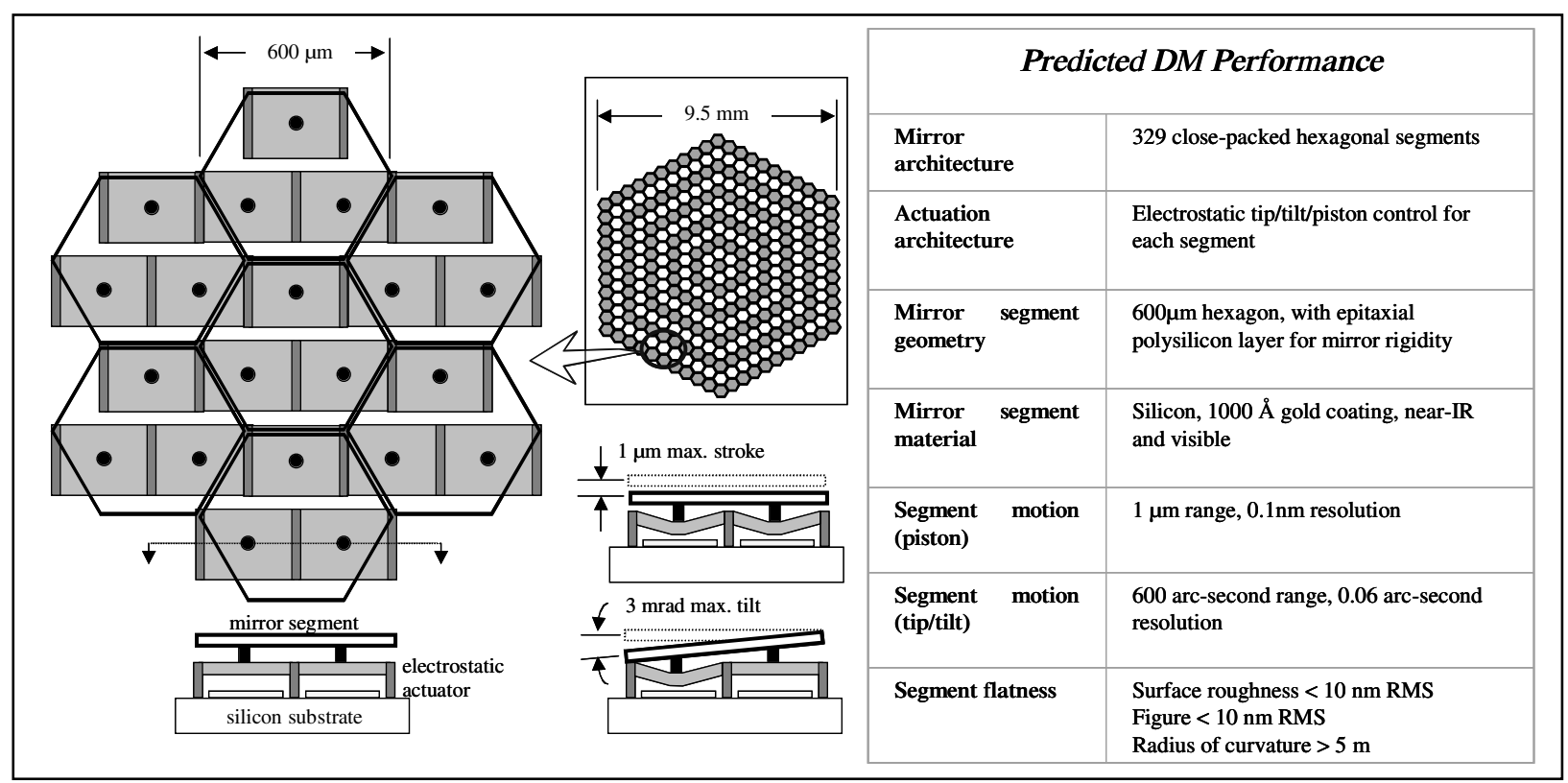

Figure 2: Top and side view of the new MEMS TPF DM architecture for tip/tilt and piston motion (left). Table summarizing predicted DM performance (right).

The microfabrication process for the TPF DM begins with a three-layer polysilicon surface micromachining process (similar to the inherited process) that involves the deposition and patterning of alternating layers of sacrificial and structural thin films (Figure 3). The $1^{\text {st }}$ and $2^{\text {nd }}$ polysilicon layers in the process developed are $500 \mathrm{~nm}$ and $2 \mathrm{I} \mathrm{m}$ thick, respectively, and the two sacrificial oxide layers are $5 \mathrm{I} \mathrm{m}$ thick. The $3^{\text {rd }}$ polycrystalline silicon layer, which used to be the final mirror surface, is $3 \mathrm{I} \mathrm{m}$ thick and it is used as a seed layer for a thicker epitaxial grown polysilicon layer. The purpose for this epi-polysilicon layer is to add rigidity to the mirror segment and aid in the chemo-mechanical polishing of the mirror surface to achieve the 10nm RMS surface roughness needed for the nulling coronagraph. Other process modifications used to improve mirror optical quality include the removal of oxide etch-access holes on the mirror surface to reduce diffraction effects, and the addition of a polishing step after the $2^{\text {nd }}$ oxide deposition to reduce printthrough from the patterned layers below.

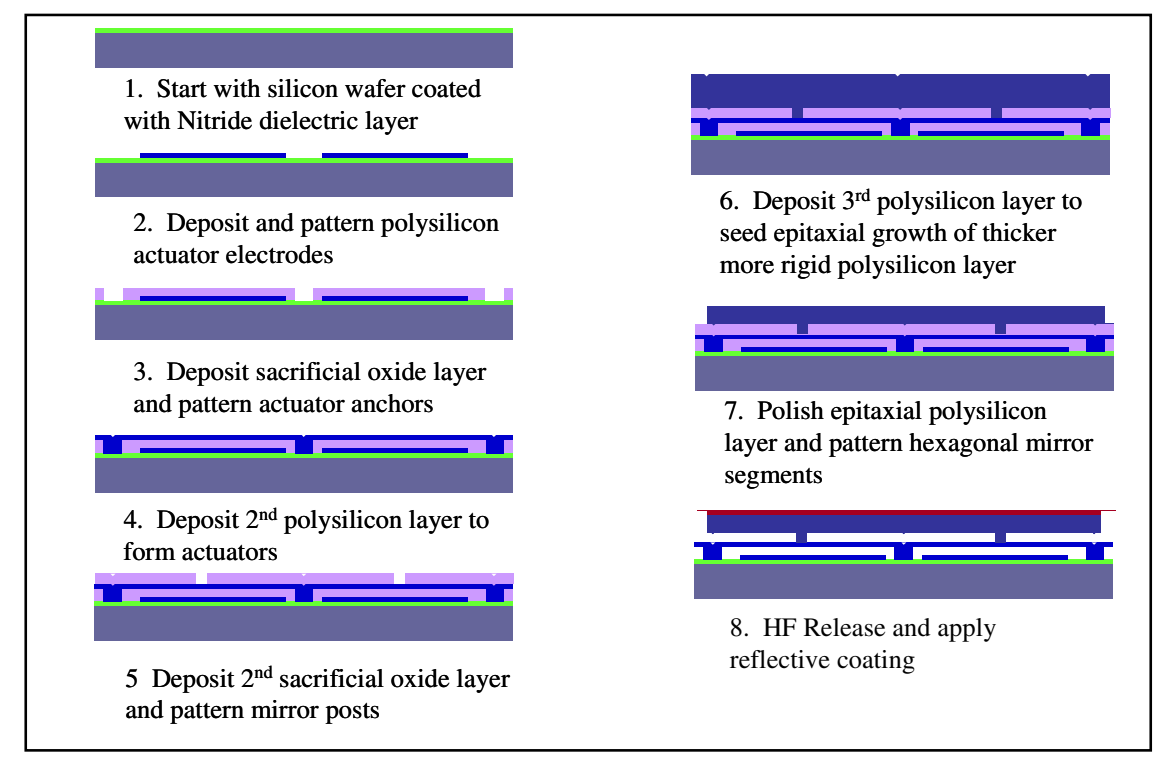

Figure 3: Profile of microfabrication process for the new TPF DM. The added layer in step \#6 combines two polysilicon deposition steps. 
The remaining mirror fabrication steps consist of the deep reactive ion etch (DRIE) of the final two polysilicon layers to partition the 329 hexagonal mirror segments (stopping in the $2^{\text {nd }}$ oxide layer), a hydrofluoric acid wet etch of the sacrificial oxide layers to release the micromirror array and a $\mathrm{CO}_{2}$ supercritical dry to prevent stiction. The mirror surface is then coated with a thin $200 \mathrm{~nm}$ layer of evaporated gold to enable broadband reflectance in the visible to nearIR.

As previously mentioned, a significant challenge in the design of the TPF DM was the development of a mirror segment that remains rigid during actuation. The nulling coronagraph specifications require the DM segments to maintain their figure to less than 10nm RMS. A thin mirror layer, such as the 3I m thick polysilicon mirror layer of the inherited DM technology, will bend during actuation due to moments applied through the actuator post attachments to the mirror segment. If the thickness of the mirror segment is increased, or if the electrostatic actuators for the segment are decoupled from the mirror surface, bending can be reduced to a level acceptable to the nulling coronagraph. Finite element modeling of the TPF DM design led to two acceptable architectures that are based on these principles. The first architecture uses conventional actuator designs and a mirror segment layer thick enough to resist bending moments introduced by the rigidly attached electrostatic actuators during tip/tilt motion. The epitaxial growth of polysilicon on the mirror surface (as described above) is capable of achieving a range of thicknesses. According to the finite element modeling results, the thickness critical to reduce mirror bending to an acceptable level was found to be about $8 \mathrm{Im}$ (Figure 4). However, to improve the likelihood of meeting the minimum nulling coronagraph optical requirements, a thickness of $15 \mathrm{I} \mathrm{m}$ was pursued.

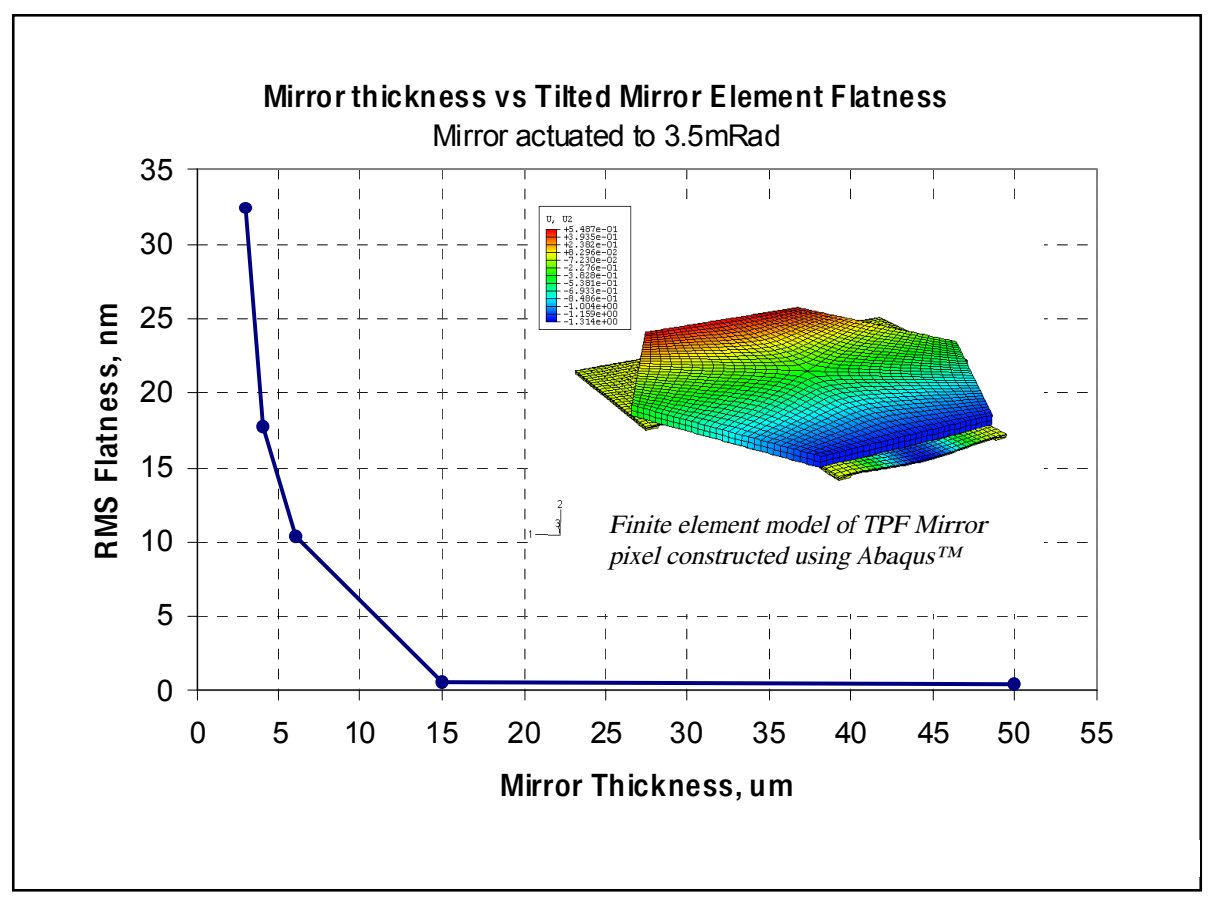

Figure 4: RMS planarity after tilting. Mirror segment bending is plotted versus epitaxial grown polysilicon thickness. Critical thickness for satisfying nulling coronagraph requirements is $15 \mathrm{I} \mathrm{m}$.

The second architecture developed decouples mirror segment tip/tilt motion from the deflection of its electrostatic actuators. This is performed by etching flexure cuts in the compliant actuator diaphragm (the $2^{\text {nd }}$ polysilicon device layer), encircling the post connection to the mirror segment, giving the mirror post freedom to move with the mirror segment as it tips and tilts. This reduces the bending moment imparted to the mirror segment by the mirror post. The flexure design is also known as the gimbal actuator design because it resembles a two-axis gimbal, as can be seen in Figure 5. 

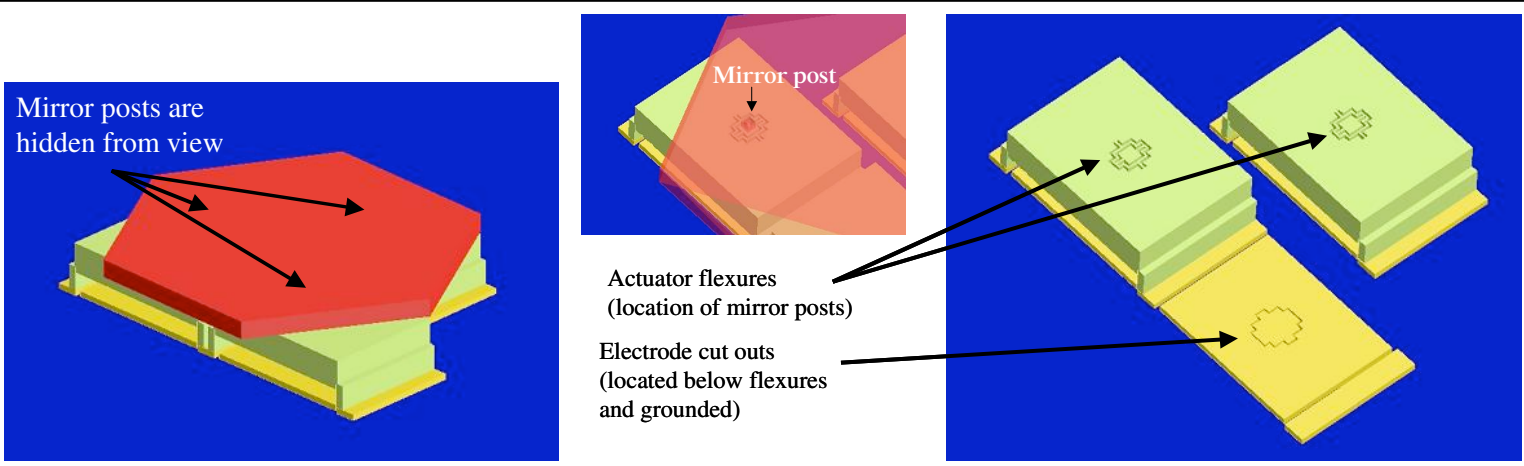

Figure 5: 3D model of the flexure (gimbal) actuator design. Full model (left), location of mirror post (center), and view of flexure cuts in actuator diaphragm with mirror segment removed (right). Designs with and without electrode alterations are currently being explored.

The results of the mirror bending finite element analysis using CoventorWare ${ }^{\mathrm{TM}}$ are displayed in Figure 6, comparing the flexure and conventional actuator designs for various mirror segment layer thicknesses. The mirror segment model was tilted along a primary tilt axis using a single actuator. For every voltage increment a cross section of the mirror was recorded and RMS flatness calculated. From these results it is apparent that for a constant mirror segment thickness (less than the critical value listed above) a substantial reduction in mirror segment bending is achieved. For example, a $3 \mathrm{I} \mathrm{m}$ thick mirror segment tilted to approximately $2 \mathrm{mrad}$ using the conventional actuator design experiences approximately $11 \mathrm{~nm}$ RMS of bending, while the flexure actuator design experiences about $1 \mathrm{~nm}$ RMS of bending, an order of magnitude improvement. Using a flexure actuator with low torsion resistance, a mirror segment thickness of approximately $4 \mathrm{I} \mathrm{m}$ would be more than sufficient to satisfy coronagraph figure requirements, compared to the $15 \mathrm{I} \mathrm{m}$ without flexure actuators. As will be discussed below, by reducing the mirror segment thickness, mirror curvature due to residual stresses becomes more manageable. A thick mirror segment that is curved due to residual stresses is more difficult to flatten via post-processing than a thinner mirror.

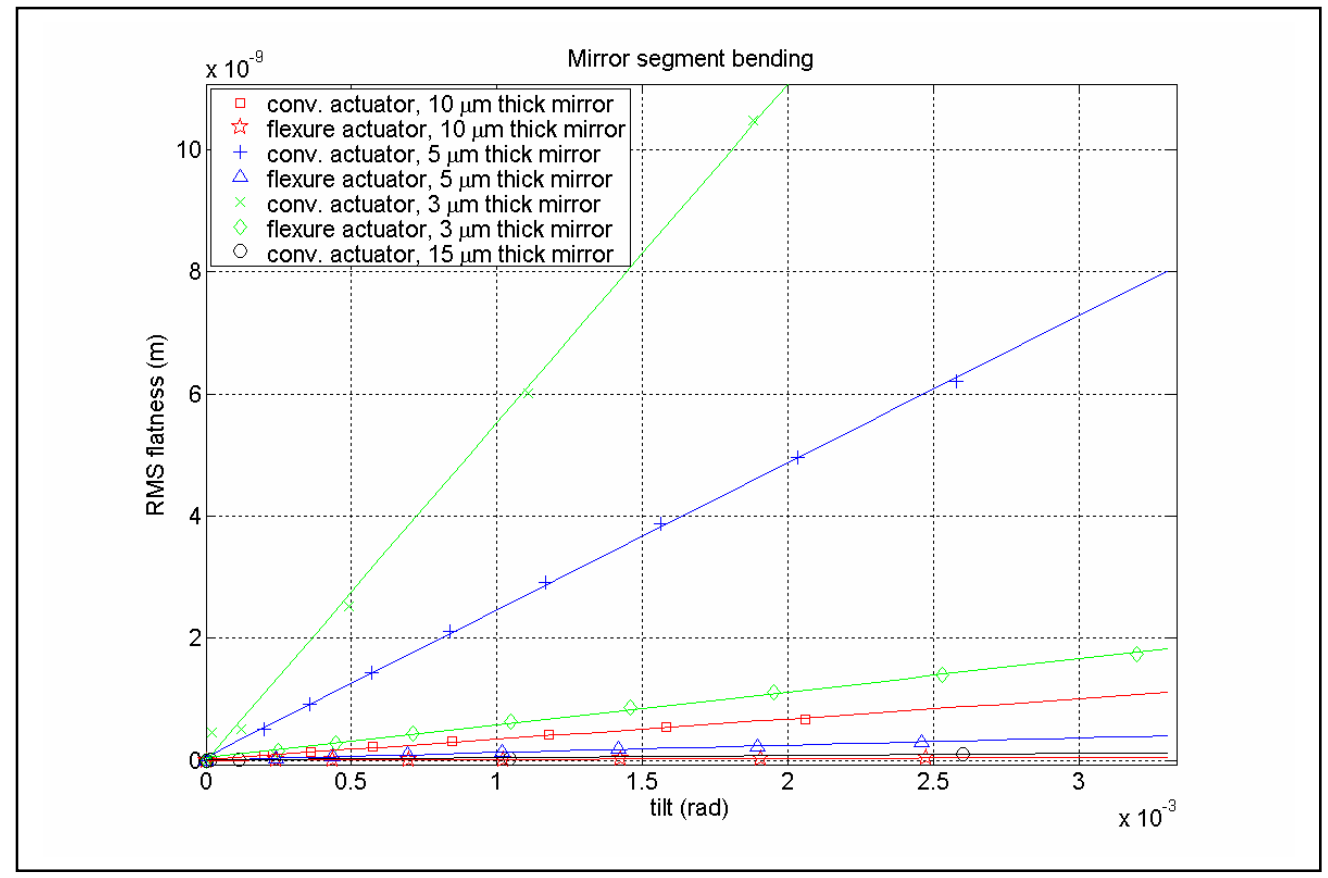

Figure 6: RMS Mirror segment bending vs. mirror segment tilt. Flexure and conventional actuator designs are compared for various mirror segment thicknesses. 


\section{DEVICE CHARACTERIZATION}

The first fabrication run for the new TPF DM consisted of several smaller 61-element arrays with 15I m and 3I m thick mirror segments (with and without the epi-polysilicon layer, respectively) using conventional electrostatic actuators. Unfortunately, the flexure actuator design was still under development at the time these mirror fabrication plans were submitted. Several 329-element arrays with flexure actuators are now in the final stages of fabrication and will be characterized at a later date. Nevertheless, the electromechanical performance (tilt motion and mirror rigidity) and optical quality of these smaller test arrays are discussed below. The fabrication of DM segments with the full 600 arcsecond range of tip/tilt motion and $1 \mathrm{I} \mathrm{m}$ of stroke was a success.

Device characterization is performed using an interferometric surface-mapping microscope that has nanometer level sensitivity. To test the electromechanical behavior of a TPF DM segment, a single actuator is energized tilting the mirror along the corresponding axis. The topography of the mirror segment is measured before and after the segment is tilted. The net motion of the mirror segment is then calculated by taking the difference between these measurements. This allows for the examination of dynamic disfigurement terms, such as mirror bending. Static mirror shapes and irregularities in the mirror surface are lost in the difference calculation, to a first order. The bending of a mirror segment upon actuation is observable when a cross section of the difference measurement is examined.

The characterization of tip/tilt behavior on multiple axes and subsequent mirror bending for six different TPF DM segments with a 3I m thick mirror segment and conventional actuators can be seen in Figure 7. This mirror architecture does not meet coronagraph flatness specifications as expected due to its $15 \mathrm{~nm}$ RMS flatness at $3 \mathrm{mrad}$ of tilt. This is in agreement with the models discussed above.
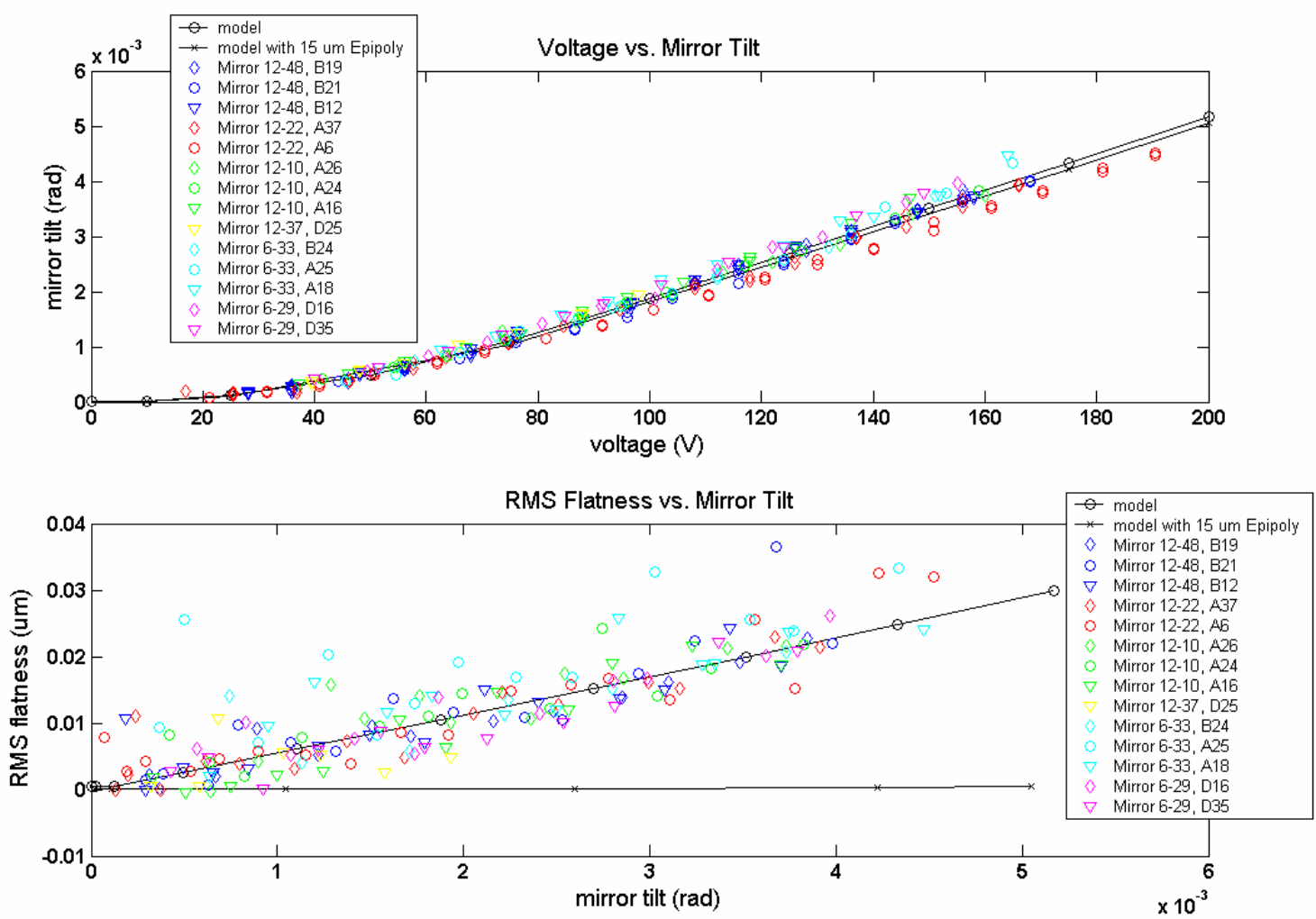

Figure 7: Electromechanical characterization of TPF DM segments that are 3I m thick and controlled using the conventional actuator design. Mirror segment flatness is greater than $15 \mathrm{~nm}$ RMS at $3 \mathrm{mrad}$ of tilt. Control voltage vs. tilt (top) and mirror segment bending vs. tilt (bottom). 
By increasing the thickness of the mirror segment to $15 \mathrm{I} \mathrm{m}$ (using the epitaxial polysilicon growth process discussed above), mirror bending is reduced to the noise level of the interference microscope, approximately 2nm RMS (Figure 8). This is a direct demonstration that the thick DM segment is capable of retaining mirror shape during tip/tilt motion, in agreement with the finite element models, and is one step closer to realizing a DM for the nulling coronagraph. These results are not affected by the piston deflection of the mirror segment.

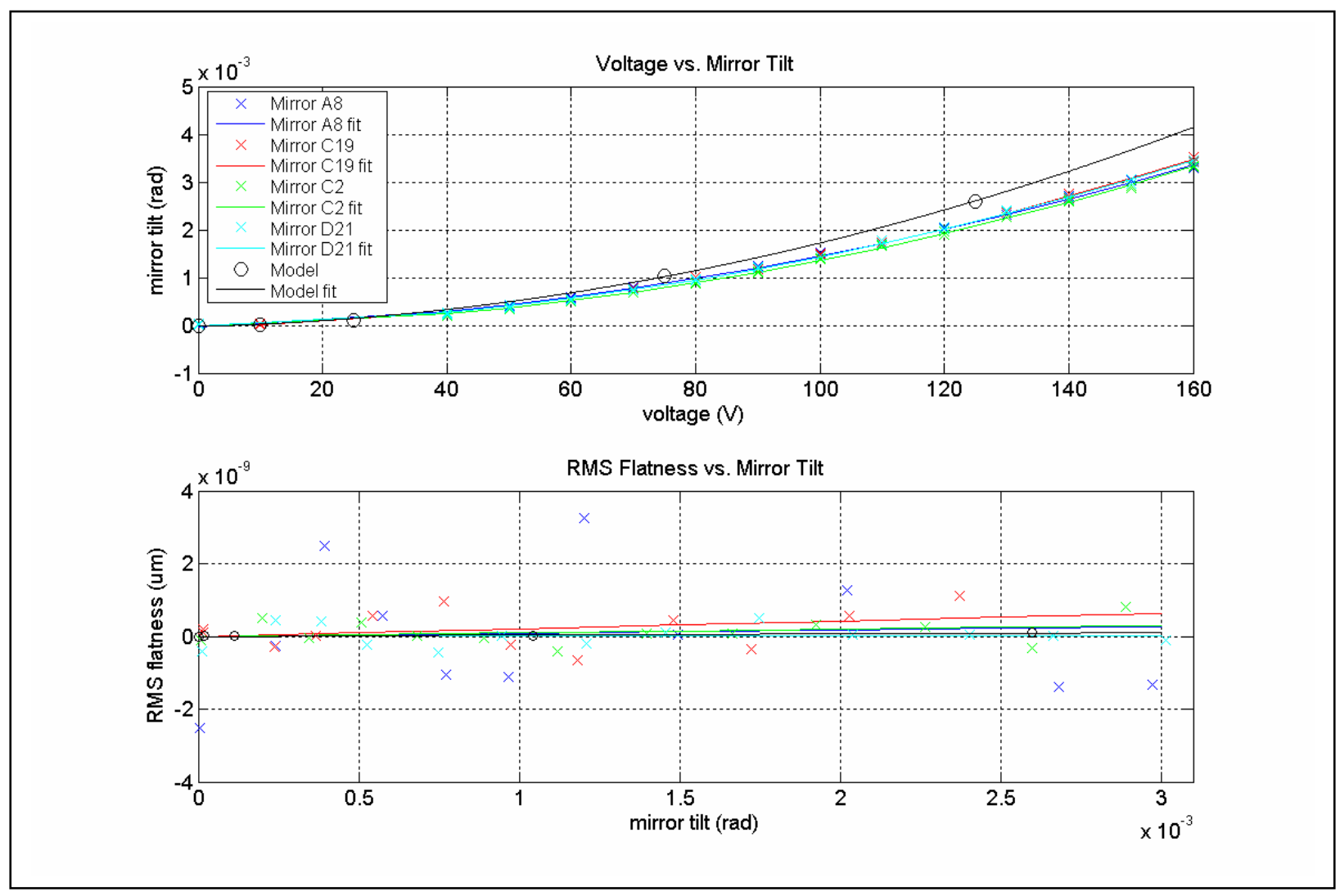

Figure 8: Electromechanical characterization of TPF DM segments that are $15 \mathrm{I}$ m thick and controlled using the conventional actuator design. Mirror segment flatness is less than $2 \mathrm{~nm}$ RMS at $3 \mathrm{mrad}$ of tilt.

Also apparent in the voltage vs. tilt data of Figure 8 (top plot) is a slight shift between model and experimental data curves. The slope of these curves is directly related to actuator stress properties. The model for mirror segment motion was first developed for the $3 \mathrm{I} \mathrm{m}$ thick mirror, and the stress in the actuator diaphragm was adjusted to match the real device behavior. This model was then used for the $15 \mathrm{I} \mathrm{m}$ thick mirror by simply changing the thickness of the mirror. The actuator stress properties remained the same. A small difference between these models is apparent in Figure 7, where they are plotted next to one another. However, the experimental data for the $15 \mathrm{I} \mathrm{m}$ thick mirror is significantly different than its model. This is evidence that the epitaxial polysilicon growth process changed the stress properties of the compliant actuator membrane, essentially increasing the spring constant of the actuator (reducing mirror tilt for equal electrostatic forces).

As discussed above, the nulling coronagraph requires that the DM segments have a radius of curvature greater than $5 \mathrm{~m}$, and that mirror segment surface roughness be less than 10nm RMS. As one can see in Figure 9, the optical quality of the polished, epitaxial grown, polysilicon DM segment is somewhere between these specifications. Due to significant tensile stress gradients in the $15 \mathrm{I} \mathrm{m}$ thick polysilicon mirror layer, the DM segments are cup shaped and have an average radius of curvature of $270 \mathrm{~mm}$. However, if the curvature of the mirror is neglected, the mirror segment has a 5nm RMS surface roughness (near the noise threshold of the interference microscope), which exceeds what is needed for the nulling coronagraph. TPF DM segments that are only 3I $\mathrm{m}$ thick, which did not have an epi-polysilicon layer deposited or polished, have mirror curvatures greater than $1 \mathrm{~m}$, but a surface roughness of 19nm RMS (primarily due to print through) 
(Figure 10). These mirrors also have conventional actuators and will bend during actuation. The polishing process used for the second oxide layer and third polysilicon (seed) layer of these mirrors is not sufficient for the nulling coronagraph optical requirements. However, a thick epitaxial grown polysilicon layer polished (and thinned) to the desired mirror segment thickness is capable of achieving the required optical surface quality, as seen with the $15 \mathrm{I} \mathrm{m}$ thick mirror. Such a process will be used in future DM fabrication runs to achieve the required surface quality.

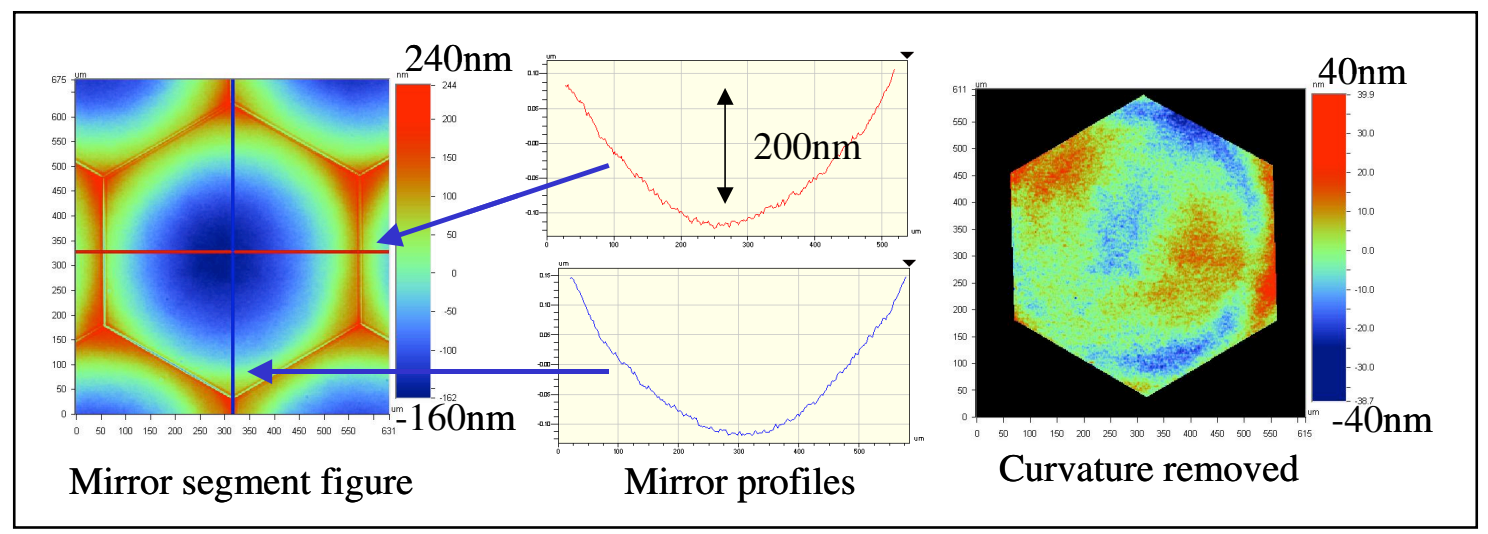

Figure 9: Surface figure of the 15I $\mathrm{m}$ thick DM segment. The radius of curvature for the mirror segments is $270 \mathrm{~mm}$ (left). Neglecting mirror curvature, the mirror segment roughness is $5 \mathrm{~nm}$ RMS (right).

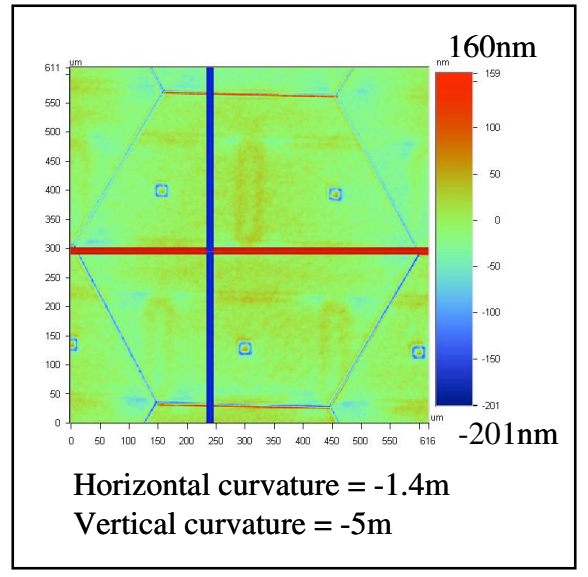

Figure 10: Surface figure for a 3I $\mathrm{m}$ thick DM segment. The radius of curvature for the mirror segments is greater than $1 \mathrm{~m}$ (pointing into the page), however without the epitaxial grown polysilicon layer, the mirror could only be polished to $12 \mathrm{~nm}$ RMS surface roughness (neglecting curvature) due to print through effects from the patterned polysilicon layers below.

The most significant task remaining for the successful fabrication of the TPF DM is the development of a method to produce flat mirror segments. A method to fine tune mirror curvature after the DM is released has been demonstrated by $\mathrm{BU}$, but it requires a thinner DM segment with tensile stress gradients. The process uses the implantation of inert gases in the mirror surface to create a thin film of compressively stressed material that acts to flatten the mirror segment ${ }^{6}$. If the flexure actuator design is successful, the final mirror segment thickness will be on the order of $4 \mathrm{I} \mathrm{m}$, which is within range of this technique. Furthermore, if the flexure actuator design succeeds, it may also be possible to fine tune tensile stress gradients through the deposition of compressively stressed thin films, such as the final reflective metal coating. Lastly, process development to reduce stress gradients in epi-polysilicon layers of various thicknesses using high temperature furnace annealing is underway. High temperature annealing of thin polysilicon films to reduce stress gradients has been successful in other MEMS applications and has been used for the production of flat DMs in the inherited BU fabrication process ${ }^{7}$. 


\section{CONCLUSION}

Realization of the TPF DM architecture will set new milestones for MEMS DMs in optical quality and micromirror control. If successfully implemented in the TPF coronagraph, this DM will become one of the first MEMS-based DMs used in a space-based astronomical observatory. DM development has overcome several technological challenges, such as a new fabrication layout to accommodate for hexagonal mirror segments with $6 \mathrm{mrad}$ tip/tilt and $1 \mathrm{I} \mathrm{m}$ piston motion, and the addition of a thicker epitaxial grown polysilicon layer to add structural rigidity to the mirror segment and aid in DM polishing to rid the mirror segments of print-through. Progress made towards the development of a DM that completely satisfies the nulling coronagraph $\Theta 100$ optical quality and electromechanical control requirements has been significant. The local surface roughness of the mirror segment is on the order of 5nm RMS, and with a 15I m thick DM segment, mirror figure is maintained to less than 2nm RMS in all degrees of freedom. A novel method to reduce DM segment bending during actuation using actuator diaphragms with flexures (resembling gimbals) has also been developed and mirror segments with these actuators will be characterized in the near future. A DM segment using flexure actuators could be thinner, making it easier to control residual stress and therefore achieve the curvature specification required by the coronagraph. The modeling results for these flexure actuators are promising.

\section{REFERENCES}

1. Terrestrial Planet Finder Web Page: http://planetquest.jpl.nasa.gov/TPF/tpf_index.cfm

2. B. M. Levine, M. Shao, D. Liu, J. K. Wallace, and B. Lane, "Planet detection in visible light with a single aperture telescope and nulling coronagraph" Proc. SPIE, 5170 (2003).

3. M. Shao, E. Serabyn, B. M. Levine, B. Mennesson, and T. Velusamy, "Visible nulling coronagraph for detecting planets around nearby stars" Proc. SPIE, 4860 (2002).

4. J. A. Perreault, T. G. Bifano, B. M. Levine, and M. Horenstein, "Adaptive optic correction using microelectromechanical deformable mirrors" Opt. Eng. 41, 561 (2002).

5. T. G. Bifano and J. B. Stewart, "High-speed wavefront control using MEMS micromirrors" Proc. SPIE, 5895 (2005).

6. T. G. Bifano, H. T. Johnson, P. Bierden, and R. K. Mali, "Elimination of stress induced curvature in thin-film structures" J. Microelectromechanical Systems 11, 592 (2002).

7. X. Zhang, T. Zhang, M. Wong, and Y. Zohar, "Rapid thermal annealing of polysilicon thin films" J. Microelectromechanical Systems 7, 356 (1998). 